\title{
Boundary Liouville Theory: Hamiltonian Description and Quantization ${ }^{\star}$
}

\author{
Harald DORN ${ }^{\dagger}$ and George JORJADZE $\ddagger$ \\ $\dagger$ Institut für Physik der Humboldt-Universität zu Berlin, \\ Newtonstraße 15, D-12489 Berlin, Germany \\ E-mail: dorn@physik.hu-berlin.de \\ $\ddagger$ Razmadze Mathematical Institute, M. Aleksidze 1, 0193, Tbilisi, Georgia \\ E-mail: jorj@rmi.acnet.ge
}

Received October 17, 2006, in final form December 11, 2006; Published online January 12, 2007

Original article is available at http://www.emis.de/journals/SIGMA/2007/012/

\begin{abstract}
The paper is devoted to the Hamiltonian treatment of classical and quantum properties of Liouville field theory on a timelike strip in $2 d$ Minkowski space. We give a complete description of classical solutions regular in the interior of the strip and obeying constant conformally invariant conditions on both boundaries. Depending on the values of the two boundary parameters these solutions may have different monodromy properties and are related to bound or scattering states. By Bohr-Sommerfeld quantization we find the quasiclassical discrete energy spectrum for the bound states in agreement with the corresponding limit of spectral data obtained previously by conformal botstrap methods in Euclidean space. The full quantum version of the special vertex operator $e^{-\varphi}$ in terms of free field exponentials is constructed in the hyperbolic sector.
\end{abstract}

Key words: Liouville theory; strings and branes; $2 d$ conformal group; boundary conditions; symplectic structure; canonical quantization

2000 Mathematics Subject Classification: 37K05; 37K30; 81T30; 81T40

\section{Introduction}

In connection with D-brane dynamics in string theory there has been a renewed interest in Liouville theory with boundaries. Within the boundary state formalism of conformal field theory the complete set of boundary states representing Dirichlet conditions (ZZ branes) [1] and generalized Neumann conditions (FZZT branes) $[2,3]$ has been constructed, including an intriguing relation between ZZ and FZZT branes [4].

While FZZT branes naturally arise in the quantization of the Liouville field with certain classical boundary conditions, the set of ZZ branes, counted by two integer numbers, so far has not found a complete classical counterpart. It seems to us an open question whether or not all ZZ branes can be understood as the quantization of a classical set up. Thus motivated we are searching for a complete treatment of the boundary Liouville theory relying only on Minkowski space Hamiltonian methods.

A lot of work in this direction has been performed already in the early eighties by Gervais and Neveu [5, 6, 7, 8], see also [9]. They restricted themselves to solutions with elliptic monodromy representing bound states of the Liouville field. Obviously such a restriction is not justified if one wants to make contact with the more recent results mentioned above. They also get

${ }^{\star}$ This paper is a contribution to the Proceedings of the O'Raifeartaigh Symposium on Non-Perturbative and Symmetry Methods in Field Theory (June 22-24, 2006, Budapest, Hungary). The full collection is available at http://www.emis.de/journals/SIGMA/LOR2006.html 
a quantization of the parameters characterizing the FZZT branes [7, 8], which is not confirmed by the more recent investigations $[2,3]$.

Our paper will be a first step in a complete Minkowski space Hamiltonian treatment of Liouville field theory on a strip with independent FZZT type boundary conditions on both sides, which for a special choice of the boundary and monodromy parameters become of ZZ type. Concerning the classical field theory, parts of our treatment will be close to the analysis of coadjoint orbits of the Virasoro algebra in [10]. As a new result we consider the assignment of these orbits to certain boundary conditions. We also give a unified treatment of all monodromies, i.e. bound states and scattering states, derive the related symplectic structure, discuss a free field parametrization and perform first steps to the quantization.

\section{Classical description}

Let us consider the Liouville equation

$$
\left(\partial_{\tau}^{2}-\partial_{\sigma}^{2}\right) \varphi(\tau, \sigma)+4 m^{2} e^{2 \varphi(\tau, \sigma)}=0
$$

on the strip $\sigma \in(0, \pi), \tau \in \mathbb{R}^{1}$, where $\sigma$ and $\tau$ are space and time coordinates, respectively. Introducing the chiral coordinates $x=\tau+\sigma, \bar{x}=\tau-\sigma$ and the exponential field $V=e^{-\varphi}$, equation (2.1) can be written as

$$
V \partial_{x \bar{x}}^{2} V-\partial_{x} V \partial_{\bar{x}} V=m^{2}, \quad V>0 .
$$

The conformal transformations of the strip are parameterized by functions $\xi(x)$, which satisfy the conditions

$$
\xi^{\prime}(x)>0, \quad \xi(x+2 \pi)=\xi(x)+2 \pi .
$$

Note that the function $\xi$ is the same for the chiral and the anti-chiral coordinates $x \mapsto \xi(x)$, $\bar{x} \mapsto \xi(\bar{x})$. This group usually is denoted by $\widetilde{\operatorname{Diff}}_{+}\left(S^{1}\right)$, since it is a covering group of the group of orientation preserving diffeomorphisms of the circle $\operatorname{Diff}_{+}\left(S^{1}\right)$.

The space of solutions of equation (2.2) is invariant under the transformations

$$
V(x, \bar{x}) \mapsto \frac{1}{\sqrt{\xi^{\prime}(x) \xi^{\prime}(\bar{x})}} V(\xi(x), \xi(\bar{x})),
$$

which is the basic symmetry of the Liouville model, and a theory on the strip has to be specified by boundary conditions invariant with respect to (2.4).

The invariance of the Dirichlet conditions

$$
\left.V\right|_{\sigma=0}=0=\left.V\right|_{\sigma=\pi}
$$

is obvious, but note that the corresponding Liouville field becomes singular $\varphi \rightarrow+\infty$ at $\sigma=0$ and $\sigma=\pi$.

Taking into account that $\xi^{\prime}(x)=\xi^{\prime}(\bar{x})$ at the boundaries, another set of invariant boundary conditions can be written in the Neumann form

$$
\left.\partial_{\sigma} V\right|_{\sigma=0}=-2 m l,\left.\quad \partial_{\sigma} V\right|_{\sigma=\pi}=2 m r,
$$

with constant boundary parameters $l$ and $r$. We study the Minkowskian case and our aim is to develop the operator approach similarly to the periodic case $[11,12,13,14,15]$. In this section we describe the conformally invariant classes of Liouville fields on the strip and give their Hamiltonian analysis; preparing, thereby, the systems for quantization. 
The energy-momentum tensor of Liouville theory

$$
T=\frac{\partial_{x x}^{2} V(x, \bar{x})}{V(x, \bar{x})}, \quad \bar{T}=\frac{\partial_{\bar{x} \bar{x}}^{2} V(x, \bar{x})}{V(x, \bar{x})}
$$

is chiral $\partial_{\bar{x}} T=0=\partial_{x} \bar{T}$. The linear combinations $T+\bar{T}$ and $T-\bar{T}$ correspond to the energy density $\mathcal{E}$ and the energy flow $\mathcal{P}$, respectively,

$$
\begin{aligned}
& \mathcal{E}=T+\bar{T}=\frac{1}{2}\left(\partial_{\tau} \varphi\right)^{2}+\frac{1}{2}\left(\partial_{\sigma} \varphi\right)^{2}+2 m^{2} e^{2 \varphi}-\partial_{\sigma \sigma}^{2} \varphi \\
& \mathcal{P}=T-\bar{T}=\partial_{\tau} \varphi \partial_{\sigma} \varphi-\partial_{\tau \sigma}^{2} \varphi .
\end{aligned}
$$

The Neumann conditions (2.6) provide vanishing energy flow at the boundaries, which leads to

$$
T(\tau)=\bar{T}(\tau) \quad \text { and } \quad T(\tau+2 \pi)=T(\tau) .
$$

The Dirichlet condition (2.5) allows ambiguities for the boundary behaviour of $T$ and $\bar{T}$. In this case we introduce the conditions (2.8) as additional to (2.5), which means that we assume regularity of $T$ and $\bar{T}$ at the boundaries and require vanishing energy flow there. Then, due to (2.7) and (2.2), the field $V$, in both cases (2.5) and (2.6), can be represented by

$$
V(x, \bar{x})=m[a \psi(\bar{x}) \psi(x)+b \psi(\bar{x}) \chi(x)+c \chi(\bar{x}) \psi(x)+d \chi(\bar{x}) \chi(x)] .
$$

Here $\psi(x), \chi(x)$ are linearly independent solutions of Hill's equation

$$
\psi^{\prime \prime}(x)=T(x) \psi(x), \quad \chi^{\prime \prime}(x)=T(x) \chi(x)
$$

with the unit Wronskian

$$
\psi(x) \chi^{\prime}(x)-\psi^{\prime}(x) \chi(x)=1,
$$

and the coefficients $a, b, c, d$ form a $S L(2, \mathbb{R})$ matrix: $a d-b c=1$. With the notations

$$
\Psi=\left(\begin{array}{c}
\psi \\
\chi
\end{array}\right), \quad \Psi^{T}=\left(\begin{array}{ll}
\psi & \chi
\end{array}\right), \quad A=\left(\begin{array}{cc}
a & b \\
c & d
\end{array}\right)
$$

equation (2.9) becomes $V(x, \bar{x})=m \Psi^{T}(\bar{x}) A \Psi(x)$. The periodicity of $T(x)$ leads to the monodromy property $\Psi(x+2 \pi)=M \Psi(x)$, with $M \in S L(2, \mathbb{R})$. The Wronskian condition (2.11) is invariant under the $S L(2, \mathbb{R})$ transformations $\Psi \mapsto S \Psi$, which transform the monodromy matrix by $M \mapsto S M S^{-1}$. A special case is $M= \pm I$, which is invariant under the $S L(2, \mathbb{R})$ maps. Otherwise $M$ can be transformed to one of the following forms [10]

$$
\begin{aligned}
& M_{h}= \pm\left(\begin{array}{cc}
e^{-\pi p} & 0 \\
0 & e^{\pi p}
\end{array}\right), \quad p>0, \quad M_{p}= \pm\left(\begin{array}{ll}
1 & 0 \\
b & 1
\end{array}\right), \quad b= \pm 1 \\
& M_{e}= \pm\left(\begin{array}{cc}
\cos \pi \theta & \sin \pi \theta \\
-\sin \pi \theta & \cos \pi \theta
\end{array}\right), \quad \theta \in(0,1),
\end{aligned}
$$

which are called hyperbolic, parabolic and elliptic monodromies, respectively. The matrices $A, M$ and the freedom related to the transformations $\Psi \mapsto S \Psi$ can be specified by the boundary conditions. First we consider the case (2.5). 


\subsection{Dirichlet condition}

The functions $\psi^{2}(\tau), \chi^{2}(\tau)$ and $\psi(\tau) \chi(\tau)$ are linearly independent and the boundary condition $\left.V\right|_{\sigma=0}=0$ with (2.9) leads to $a=0=d=b+c$. Then, we find

$$
V(x, \bar{x})=m[\psi(\bar{x}) \chi(x)-\chi(\bar{x}) \psi(x)],
$$

which corresponds to $b=1=-c$. Note that the behaviour of (2.13) near to the boundary $\sigma \sim \epsilon$ is given by $V=2 m \epsilon+O\left(\epsilon^{3}\right)$, the other choice $c=1=-b$ corresponds to negative $V$ near to $\sigma=0$ and has to be neglected. Applying the boundary condition $\left.V\right|_{\sigma=\pi}=0$ to (2.13) and using the monodromy property we obtain $\Psi(x+2 \pi)= \pm \Psi(x)$. Expanding $V$ now near to the right boundary $\sigma \sim \pi-\epsilon$, we get $V=\mp 2 m \epsilon+O\left(\epsilon^{3}\right)$. Thus, the allowed monodromy is $\Psi(x+2 \pi)=-\Psi(x)$ only. After fixing the matrices $A$ and $M$ we have to specify the class of functions $\psi$ and $\chi$, which ensures the positivity of $V$ in the whole bulk $\sigma \in(0, \pi)$.

Representing $(\psi, \chi)$ in polar coordinates, due to the unit Wronskian condition, the radial coordinate is fixed in terms of the angle variable $\xi(x) / 2$ resulting in

$$
\psi(x)=\sqrt{\frac{2}{\xi^{\prime}(x)}} \cos \frac{\xi(x)}{2}, \quad \chi(x)=\sqrt{\frac{2}{\xi^{\prime}(x)}} \sin \frac{\xi(x)}{2},
$$

and by (2.13) the $V$-field becomes

$$
V=\frac{2 m}{\sqrt{\xi^{\prime}(x) \xi^{\prime}(\bar{x})}} \sin \frac{1}{2}[\xi(x)-\xi(\bar{x})] .
$$

The obtained monodromy of $\Psi(x)$ leads to $\xi(x+2 \pi)=\xi(x)+2 \pi(2 n+1)$ with arbitrary integer $n$, but (2.15) is positive in the whole strip $\sigma \in(0, \pi)$ for $n=0$ only. Thus, $\xi(x)$ turns out to be just a function parameterizing a diffeomorphism according to (2.3). Equation (2.13) is invariant under the $S L(2, \mathbb{R})$ transformations $\Psi \mapsto S \Psi$. The corresponding infinitesimal form of $\xi(x)$ is

$$
\xi(x) \mapsto \xi(x)+\varepsilon_{1}+\varepsilon_{2} \cos \xi(x)+\varepsilon_{3} \sin \xi(x)
$$

and the space of solutions $(2.15)$ is identified with $\widetilde{\operatorname{Diff}_{+}}\left(S^{1}\right) / \widetilde{S L}(2, \mathbb{R})$.

The energy momentum tensor (2.7) calculated from (2.15) reads

$$
T(x)=-\frac{1}{4} \xi^{\prime 2}(x)+S_{\xi}(x), \quad \text { with } \quad S_{\xi}(x)=\left(\frac{\xi^{\prime \prime}(x)}{2 \xi^{\prime}(x)}\right)^{2}-\left(\frac{\xi^{\prime \prime}(x)}{2 \xi^{\prime}(x)}\right)^{\prime}
$$

and for $\xi(x)=x$ it is constant $T=-\frac{1}{4}$. The corresponding Liouville field is time-independent

$$
\varphi_{0}=-\log (2 m \sin \sigma),
$$

and it is associated with the vacuum of the system. The vacuum solution is invariant under the $S L(2, \mathbb{R})$ subgroup of conformal transformations generated by the vector fields $\partial_{x}, \cos x \partial_{x}$ and $\sin x \partial_{x}$ and this symmetry is a particular case of $(2.16)$ for $\xi(x)=x$. Therefore, the solutions of the Liouville equation with Dirichlet boundary conditions form the conformal orbit of the vacuum solution (2.18). The energy functional

$$
E=\int_{0}^{\pi} d \sigma(T(\tau+\sigma)+T(\tau-\sigma))=\int_{0}^{2 \pi} d x T(x)
$$

on this orbit is bounded below and the minimal value is achieved for the vacuum configuration [10]. 
To get the Hamiltonian description we first specify the boundary behaviour of Liouville fields. Due to (2.10), (2.11), (2.13), near to the boundaries $V$ is given by

$$
V=2 m \epsilon+\frac{4 m}{3} T(\tau) \epsilon^{3}+O\left(\epsilon^{5}\right)
$$

and we find

$$
\begin{array}{ll}
\varphi=-\log 2 m \epsilon-\frac{2}{3} T \epsilon^{2}+O\left(\epsilon^{4}\right), & \partial_{\tau} \varphi=-\frac{2}{3} \dot{T} \epsilon^{2}+O\left(\epsilon^{4}\right), \\
\partial_{\sigma} \varphi= \pm\left(\frac{1}{\epsilon}+\frac{4}{3} T \epsilon\right)+O\left(\epsilon^{3}\right), & \partial_{\sigma \sigma}^{2} \varphi=\frac{1}{\epsilon^{2}}-\frac{4}{3} T+O\left(\epsilon^{2}\right) .
\end{array}
$$

The signs + and - for $\partial_{\sigma} \varphi$ correspond to the right $(\sigma=\pi)$ and left $(\sigma=0)$ boundaries and the argument of $T$ is $\tau+\pi$ and $\tau$, respectively. The Liouville equation (2.1) is equivalent to the Hamilton equations obtained from the canonical action

$$
S=\int d \tau \int_{0}^{\pi} d \sigma\left[\pi \dot{\varphi}-\left(\frac{1}{2} \pi^{2}+\frac{1}{2}\left(\partial_{\sigma} \varphi\right)^{2}+2 m^{2} e^{2 \varphi}-\partial_{\sigma \sigma}^{2} \varphi\right)\right]
$$

with the Hamiltonian given by the energy functional (2.19). Note that $\partial_{\sigma \sigma}^{2} \varphi$ can not be integrated into a boundary term due to the singularities (2.21).

The canonical 2 -form related to $(2.22)$

$$
\omega=\int_{0}^{\pi} d \sigma \delta \pi(\tau, \sigma) \wedge \delta \varphi(\tau, \sigma)
$$

is well defined on the class of singular functions (2.20) and using the parameterization (2.13)(2.14), we find this 2 -form in terms of the $\xi$-field (see Appendix A)

$$
\omega=\frac{1}{4} \int_{0}^{2 \pi} d x\left[\frac{\delta \xi^{\prime \prime}(x) \wedge \delta \xi^{\prime}(x)}{\xi^{\prime 2}(x)}-\delta \xi^{\prime}(x) \wedge \delta \xi(x)\right] .
$$

It is degenerated, but has to be reduced on the space $\widetilde{\operatorname{Diff}_{+}}\left(S^{1}\right) / \widetilde{S L}(2, \mathbb{R})$, where it becomes symplectic.

One can of course also study the case with Dirichlet conditions on one and Neumann conditions on the other boundary of the strip, say

$$
\left.V\right|_{\sigma=0}=0,\left.\quad \partial_{\sigma} V\right|_{\sigma=\pi}=2 m r .
$$

Starting again with (2.13), the boundary condition at $\sigma=\pi$, together with the unit Wronskian forces the trace of the monodromy matrix to be equal to $2 r$. Thus for $|r|<1$ one has elliptic and for $r>1$ hyperbolic monodromy ( $r<-1$ is excluded by arguments of the next section). Then an analysis similar to the one above gives for $-1 \leq r<1$

$$
V=\frac{2 m}{\theta \sqrt{\xi^{\prime}(x) \xi^{\prime}(\bar{x})}} \sin \frac{\theta}{2}[\xi(x)-\xi(\bar{x})]
$$

with $r=\cos \pi \theta$. The related energy momentum tensor is

$$
T(x)=-\frac{\theta^{2}}{4} \xi^{\prime 2}(x)+S_{\xi}(x) .
$$

The result for $r>1$ is obtained by the replacement $\theta=i p$ with real $p$. 


\subsection{Generalized Neumann conditions}

To analyze the Neumann conditions (2.6) we first construct the fields corresponding to constant energy-momentum tensor $T(x)=T_{0}$ and then obtain others by conformal transformations. It appears that this construction covers all regular Liouville fields on the strip.

For $T_{0}=p^{2} / 4>0$ convenient solutions of Hill's equation are

$$
\psi(x)=\cosh (p x / 2), \quad \chi(x)=\frac{2}{p} \sinh (p x / 2) .
$$

The related monodromy is hyperbolic. To get it in the normal form (2.12) one has to switch to corresponding exponentials. The form chosen allows a smooth limit for $\psi$ and $\chi$ to the parabolic and elliptic cases below. The corresponding $V$-field (2.9), which obeys the Neumann conditions (2.6), reads

$$
V_{0}(x, \bar{x})=\frac{2 m}{p \sinh \pi p}\left(u \cosh p\left(\tau-\tau_{0}\right)+l \cosh p(\sigma-\pi)+r \cosh p \sigma\right),
$$

where

$$
u=u(l, r ; p)=\sqrt{l^{2}+r^{2}+2 l r \cosh \pi p+\sinh ^{2} \pi p},
$$

and $\tau_{0}$ is an arbitrary constant. The positivity of the $V$-field (2.25) imposes restrictions on the parameters of the theory. One can show that if $l<-1$ or $r<-1$, then the positivity of (2.25) fails for any $p>0$; but if $l \geq-1$ and $r \geq-1$, then (2.25) is positive in the whole bulk $\sigma \in(0, \pi)$ for all $p>0$.

For $T_{0}=0$ we can choose $\psi(x)=1, \chi(x)=x$ and obtain

$$
V_{0}=\frac{m}{\pi}\left[(l+r)\left(\sigma^{2}-\left(\tau-\tau_{0}\right)^{2}\right)-\pi l(2 \sigma-\pi)-\pi^{2} \frac{1+l r}{l+r}\right] .
$$

This case corresponds to the parabolic monodromy. The positivity of (2.27) requires $l \geq-1$, $r \geq-1$ and $l+r<0$. Note that (2.27) is also obtained from (2.25) in the limit $p \rightarrow 0$, if $l+r<0$. Among these parabolic solutions there are two time-independent solutions

$$
V_{0}=2 m \sigma \quad \text { and } \quad V_{0}=2 m(\pi-\sigma),
$$

which correspond to the degenerated cases of (2.27) for $l=-1, r=1$ and $l=1, r=-1$, respectively.

For $T_{0}=-\theta^{2} / 4<0$ the pair

$$
\psi(x)=\cos (\theta x / 2), \quad \chi(x)=\frac{2}{\theta} \sin (\theta x / 2),
$$

has elliptic monodromy and the $V$-field becomes

$$
V_{0}=-\frac{2 m}{\theta \sin \pi \theta}\left(u \cos \theta\left(\tau-\tau_{0}\right)+l \cos \theta(\sigma-\pi)+r \cos \theta \sigma\right),
$$

with $u=u(l, r ; i \theta)=\sqrt{l^{2}+r^{2}+2 l r \cos \pi \theta-\sin ^{2} \pi \theta}$. Due to oscillations in $\sigma$ the positivity of (2.28) in the whole bulk fails if $\theta>1$; hence, $\theta \leq 1$.

Other restrictions on the parameters come from the analysis of the equation

$$
l^{2}+r^{2}+2 l r \cos \pi \theta-\sin ^{2} \pi \theta=0,
$$

which defines an ellipse on the $(l, r)$-plane. The ellipse is centered at the origin, its half axis with length $\sqrt{1 \pm \cos \pi \theta}$ are situated on the lines $r \pm l=0$. It is tangential to the lines $l=-1$ 


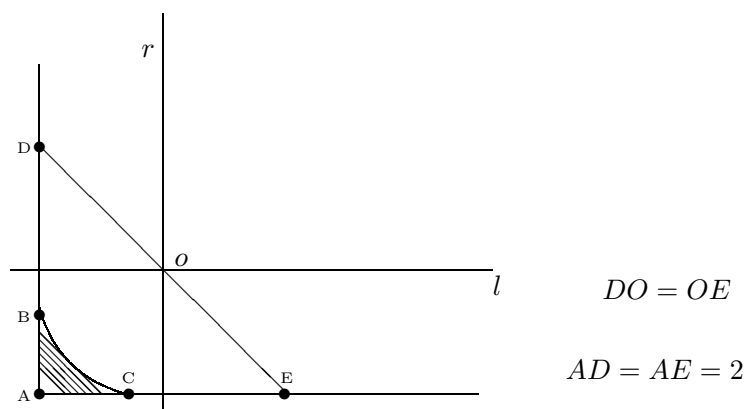

Figure 1.

and $r=-1$ at the points $B$ and $C$ with the coordinates $B=(-1, \cos \pi \theta)$ and $C=(\cos \pi \theta,-1)$. The curve $B C$ in Fig. 1 indicates the corresponding arc of the ellipse. The positivity of $V$-field (2.28) requires $(l, r) \in \Omega_{A B C}$, where $\Omega_{A B C}$ is the 'triangle' bounded by the lines $l=-1, r=-1$ and the arc $B C$.

Having so far discussed which values of $l$ and $r$ are allowed for given $\theta$, we now turn the question around. We start with $(l, r)$ somewhere in the triangle $A D E$, then all $\theta$ obeying

$$
0 \leq \theta \leq \theta_{*}(l, r)
$$

with $\theta_{*}(l, r)$ defined by

$$
\cos \pi \theta_{*}=-l r+\sqrt{\left(1-l^{2}\right)\left(1-r^{2}\right)}, \quad 0<\theta_{*} \leq 1 .
$$

are allowed ${ }^{1}$. For $\theta=\theta_{*}$ the $V$-field is time-independent

$$
V_{0}=-\frac{2 m}{\theta_{*} \sin \pi \theta_{*}}\left[l \cos \theta_{*}(\sigma-\pi)+r \cos \theta_{*} \sigma\right] .
$$

For $\theta_{*}=1$, the arc $B C$ degenerates to the point $A$ and the Liouville field (2.31) becomes the Dirichlet (ZZ) vacuum (2.18). Thus, for the critical values of the boundary parameters $l=r=-1$ the Neumann (FZZT) case contains the $Z Z$ case.

The above analysis shows that the admissible values of the boundary parameters are $l \geq-1$, $r \geq-1$ and for a given $(l, r)$ obeying these constraints there are the following restrictions on $T_{0}$

$$
\begin{array}{llll}
l+r>0 & & \Rightarrow & T_{0}>0, \\
l+r=0 \quad \text { and } \quad l \neq \pm 1 & \Rightarrow & T_{0}>0, \\
l+r=0 \quad \text { and } \quad l= \pm 1 & \Rightarrow & T_{0} \geq 0, \\
l+r<0 & & & \\
l & & T_{0} \geq-\frac{\theta_{*}^{2}(l, r)}{4} .
\end{array}
$$

Thus, we have all three monodromies if $(l, r)$ is inside the triangle $A D E$, and only hyperbolic monodromy if $(l, r)$ is outside of it. In the first case (2.28) is a continuation of (2.25) from positive to negative $T_{0}$ and for $T_{0}=0$ it coincides with (2.27).

The conformal orbits, generated out of these fields with constant $T(x)=T_{0}$ can be written as

$$
\begin{aligned}
V(x, \bar{x})= & \frac{m\left(\xi^{\prime}(x) \xi^{\prime}(\bar{x})\right)^{-\frac{1}{2}}}{p \sinh \pi p}\left(u(l, r ; p) e^{-(\xi(x)+\xi(\bar{x})) p / 2}+u(l, r ; p) e^{(\xi(x)+\xi(\bar{x})) p / 2}\right. \\
& \left.+\left(l e^{-\pi p}+r\right) e^{(\xi(x)-\xi(\bar{x})) p / 2}+\left(l e^{\pi p}+r\right) e^{(\xi(\bar{x})-\xi(x)) p / 2}\right),
\end{aligned}
$$

where $p=2 \sqrt{T_{0}}$ and $\xi(x)$ is the group parameter on the orbits. The parameter $\tau_{0}$ is absorbed by the zero mode of $\xi(x)$.

\footnotetext{
${ }^{1}$ Note that (2.30) is the larger root of equation (2.29) for $\cos \pi \theta$. The smaller root corresponds to the case when the point $(l, r)$ is on the ellipse but does not belong to the arc $B C$.
} 
The energy-momentum tensor for (2.32) is given by

$$
T(x)=T_{0} \xi^{\prime 2}(x)+S_{\xi}(x) .
$$

Here $S_{\xi}(x)$ is the Schwarz derivative (2.17), which defines the inhomogeneous part of the transformed $T(x)$. Note that there are other orbits, either with $T_{0}<-\frac{1}{4}$, or those, which do not contain orbits with constant $T(x)$ [10]. Using the classification of Liouville fields by coadjoint orbits, one can show that the orbits (2.32) with $T_{0} \geq-\frac{1}{4}$ cover all regular Liouville fields on the strip. One can also prove that the energy functional (2.19) is bounded below just on these orbits only [10]. Thus, boundary Liouville theory selects the class of fields with bounded energy functional and, therefore, its quantum theory should provide highest weight representations of the Virasoro algebra.

The Hamiltonian approach based on the action (2.22) is applicable for the Neumann conditions as well. Indeed, the boundary conditions (2.6) are equivalent to

$$
\left.\left(\partial_{\sigma} \varphi-2 m l e^{\varphi}\right)\right|_{\sigma=0}=0=\left.\left(\partial_{\sigma} \varphi+2 m r e^{\varphi}\right)\right|_{\sigma=\pi}
$$

and its variation yields $\partial_{\sigma}(\delta \varphi)-\left.\left(\partial_{\sigma} \varphi\right) \delta \varphi\right|_{\sigma=0, \pi}=0$, which cancels the boundary term for the variation of (2.22). The Legendre transformation of (2.22) and the integration of $\partial_{\sigma \sigma}^{2} \varphi$ into the boundary terms leads to the action [2]

$$
S=\int d \tau \int_{0}^{\pi} d \sigma \frac{1}{2}\left[\left(\partial_{\tau} \varphi\right)^{2}-\left(\partial_{\sigma} \varphi\right)^{2}-4 m^{2} e^{2 \varphi}\right]-2 m \int d \tau\left[l e^{\varphi(\tau, 0)}+r e^{\varphi(\tau, \pi)}\right] .
$$

Note that for $l=-1$ or/and $r=-1$ the $V$-field vanishes at the boundary for $\tau=\tau_{0}$ and one can not pass to (2.34), due to the singularities of $e^{\varphi}$.

The canonical 2 -form $(2.23)$ can be calculated in the variables $\left(T_{0}, \xi\right)$ similarly to $(2.24)$ and we obtain (see Appendix A)

$$
\omega=\delta T_{0} \wedge \int_{0}^{2 \pi} d x \xi^{\prime}(x) \delta \xi(x)+T_{0} \int_{0}^{2 \pi} d x \delta \xi^{\prime}(x) \wedge \delta \xi(x)+\frac{1}{4} \int_{0}^{2 \pi} d x \frac{\delta \xi^{\prime \prime}(x) \wedge \delta \xi^{\prime}(x)}{\xi^{\prime 2}(x)}
$$

In the context of Liouville theory this symplectic form was discussed in [16], where it was obtained as a generalization of the symplectic form on the co-adjoint orbits of the $2 d$ conformal group. Note that the form of (2.35) does not depend on the boundary parameters $l$ and $r$. This dependence implicitly is encoded in the domain of $T_{0}$ : if $(l, r)$ is inside the triangle $A D E$, then $T_{0} \geq-\theta_{*}^{2},(2.30)$; and $T_{0}>0$ if $(l, r)$ is outside the triangle. The symplectic form (2.35) provides the following Poisson brackets

$$
\left\{T_{0}, \xi(x)\right\}=\frac{1}{2 \pi}, \quad\{\xi(x), \xi(y)\}=\frac{1}{4 T_{0}}\left(\frac{\sinh \left(2 \sqrt{T_{0}} \lambda(x, y)\right)}{\sinh \left(2 \pi \sqrt{T_{0}}\right)}-\frac{\lambda(x, y)}{\pi}\right),
$$

where $\lambda(x, y)=\xi(x)-\xi(y)-\pi \epsilon(x-y)$ and $\epsilon(x)$ is the stair-step function: $\epsilon(x)=2 n+1$, for $x \in(2 \pi n, 2 \pi n+2 \pi)$, which is related to the periodic $\delta$-function by $\epsilon^{\prime}(x)=2 \delta(x)$.

From (2.36) we obtain

$$
\begin{aligned}
& \{T(x), \xi(y)\}=\xi^{\prime}(x) \delta(x-y), \\
& \{T(x), T(y)\}=T^{\prime}(y) \delta(x-y)-2 T(y) \delta^{\prime}(x-y)+\frac{1}{2} \delta^{\prime \prime \prime}(x-y),
\end{aligned}
$$

which define the conformal transformations for the fields $\xi(x)$ and $T(x)$.

Using the Fourier mode expansion for $\xi(x)$

$$
\xi(x)=x+\sum_{n \in Z} \xi_{n} e^{-i n x}
$$


and the first equation of (2.36), we find that $2 \pi \xi_{0}$ is the canonical conjugated to $T_{0}$

$$
\left\{T_{0}, 2 \pi \xi_{n}\right\}=\delta_{n 0}
$$

For $T_{0}<0$ the variable $\alpha=2 \sqrt{-T_{0}} \xi_{0}$ is cyclic $(\alpha \sim \alpha+2 \pi)$, since the exponentials in (2.32) become oscillating. By (2.37), $\alpha$ is canonical conjugated to $\pi \theta$, where $\theta=2 \sqrt{-T_{0}}$.

This allows a remarkable first conclusion concerning quantization. Semi-classical BohrSommerfeld quantization of $\theta$ yields $\theta_{n}=-\hbar n / \pi+\theta_{*}(l, r)$, which implies a quantization of $T_{0}$

$$
\left(T_{0}\right)_{n}=-\frac{1}{4} \theta_{n}^{2}=-\frac{\hbar^{2}}{4 \pi^{2}} n^{2}+\frac{\hbar}{2 \pi} \theta_{*}(l, r) n-\frac{1}{4} \theta_{*}^{2},
$$

with integer $n$ as long as $\left(T_{0}\right)_{n}<0$. As shown in Appendix $\mathrm{B}$, this spectrum with the identification $\hbar=2 \pi b^{2}$ and the trivial shifts $n^{2} \mapsto n(n+1),\left(T_{0}\right)_{n} \mapsto\left(T_{0}\right)_{n}+1 / 4$ agrees with the quasiclassical expansion of the spectrum derived in [3] by highly different methods.

After this short aside we start preparing for the full quantization of our system. The variables $(\xi, p)$ are not suitable for this purpose due to the complicated form of the Poisson brackets (2.36). A natural approach in this direction is a free-field parameterization with a perspective of canonical quantization.

For $T_{0}=p^{2} / 4>0$, free-field variables can be introduced similarly to the periodic case [15]

$$
\phi(x)=\frac{p \xi(x)}{2}+\frac{1}{2} \log \xi^{\prime}(x)-\frac{1}{2} \log \frac{m u(l, r ; p)}{p \sinh \pi p} .
$$

Here the $x$-independent part given by the last term is chosen for further convenience, for $u(l, r ; p)$ see (2.26). The field $\phi(x)$ obviously has the monodromy $\phi(x+2 \pi)=\phi(x)+\pi p$, which allows the mode expansion

$$
\phi(x)=\frac{q}{2 \pi}+\frac{p x}{2}+\frac{i}{\sqrt{4 \pi}} \sum_{n \neq 0} \frac{a_{n}}{n} e^{-i n x} .
$$

The integration of (2.39) yields

$$
\xi(x)=\frac{1}{p} \log \frac{m u A_{p}(x)}{2 \sinh ^{2} \pi p},
$$

where $A_{p}(x)$ is the integral of the equation $A_{p}^{\prime}(x)=2 \sinh \pi p e^{2 \phi(x)}$ with the monodromy property $A_{p}(x+2 \pi)=e^{2 \pi p} A_{p}(x)$ and it can be written as

$$
A_{p}(x)=\int_{0}^{2 \pi} d y e^{2 \phi(x+y)-\pi p}
$$

The free-field form of (2.35)

$$
\omega=\int_{0}^{2 \pi} d x \delta \phi^{\prime}(x) \wedge \delta \phi(x)+\delta p \wedge \delta \phi(0)=\delta p \wedge \delta q+\frac{1}{2 i} \sum_{n \neq 0} \frac{1}{n} \delta a_{n} \wedge \delta a_{-n},
$$

follows from the direct computation and it provides the canonical brackets

$$
\{\phi(x), \phi(y)\}=\frac{1}{4} \epsilon(x-y), \quad \text { or } \quad\{p, q\}=1, \quad\left\{a_{n}, a_{m}\right\}=i n \delta_{n+m, 0} .
$$

Note that these brackets and (2.40) lead to (2.36).

The energy-momentum (2.33) takes also a free-field form with a linear improvement term

$$
T(x)=\phi^{\prime 2}(x)-\phi^{\prime \prime}(x),
$$


and by (2.41) we have

$$
\left\{T(x), \phi^{\prime}(y)\right\}=\phi^{\prime \prime}(y) \delta(x-y)-\phi^{\prime}(y) \delta^{\prime}(x-y)+1 / 2 \delta^{\prime \prime}(x-y) .
$$

Inserting (2.40) into (2.32), we find

$$
V=e^{-[\phi(x)+\phi(\bar{x})]}\left[1+m b_{p} A_{p}(x)+m c_{p} A_{p}(\bar{x})+m^{2} d_{p} A_{p}(x) A_{p}(\bar{x})\right],
$$

with

$$
b_{p}=\frac{l e^{-\pi p}+r}{2 \sinh ^{2} \pi p}, \quad c_{p}=\frac{l e^{\pi p}+r}{2 \sinh ^{2} \pi p}, \quad d_{p}=\frac{u^{2}(l, r ; p)}{4 \sinh ^{4} \pi p} .
$$

The field $\Phi=\phi(x)+\phi(\bar{x})$ is the full free-field on the strip. It satisfies for all allowed values of $l$ and $r$ the standard Neumann boundary conditions $\left.\partial_{\sigma} \Phi\right|_{\sigma=0}=0=\left.\partial_{\sigma} \Phi\right|_{\sigma=\pi}$ and has the following mode expansion

$$
\Phi(\tau, \sigma)=\frac{q}{\pi}+p \tau+\frac{i}{\sqrt{\pi}} \sum_{n \neq 0} \frac{a_{n}}{n} e^{-i n \tau} \cos n \sigma,
$$

Since $p>0, A_{p}(x)$ and $A_{p}(\bar{x})$ vanish for $\tau \rightarrow-\infty$. Therefore $\Phi(\tau, \sigma)$ is the $i n$-field for the Liouville field: $\varphi(\tau, \sigma) \rightarrow \Phi(\tau, \sigma)$, for $\tau \rightarrow-\infty$.

The chiral out-field is introduced similarly to (2.39) replacing $p$ by $-p$ and its mode expansion can be written as

$$
\phi_{\text {out }}(x)=\frac{\tilde{q}}{2 \pi}-\frac{p x}{2}+\frac{i}{\sqrt{4 \pi}} \sum_{n \neq 0} \frac{\tilde{a}_{n}}{n} e^{-i n x},
$$

The relation between in and out fields

$$
\phi_{\text {out }}(x)=\phi(x)-\log \frac{m u A_{p}(x)}{2 \sinh ^{2} \pi p},
$$

defines a canonical map between the modes $\left(p, q ; a_{n}\right)$ and $\left(\tilde{q},-p, \tilde{a}_{n}\right)$. Quantum mechanically this map is given by the $S$-matrix and finding its closed form is one of the basic open problems of Liouville theory.

\section{Canonical quantization}

In this section we consider canonical quantization applying the technique developed for the periodic case [11, 12, 13, 14, 15]. Our discussion has some overlap with [9]. But in contrast to their parametrization in terms of two related free fields we use only one parametrizing free field. We mainly treat the hyperbolic case. The quantum theory of other sectors can be obtained by analytical continuation in the zero mode $p$, choosing appropriate values of the boundary parameters $(l, r)$.

The canonical commutation relations

$$
[q, p]=i \hbar, \quad\left[a_{m}, a_{n}^{*}\right]=\hbar m \delta_{m n} \quad(m>0, n>0)
$$

are equivalent to the chiral commutator

$$
[\phi(x), \phi(y)]=-\frac{i \hbar}{4} \epsilon(x-y)
$$


and have a standard realization in the Hilbert space $L^{2}\left(\mathbb{R}_{+}\right) \otimes \mathcal{F}$, where $L^{2}\left(\mathbb{R}_{+}\right)$corresponds to the momentum representation of the zero modes with $p>0$ and $\mathcal{F}$ stands for the Fock space of the non-zero modes $a_{n}$. We use the same notations for classical and corresponding normal ordered quantum expressions, which, in general, have to be deformed in order to preserve the symmetries of the theory. The guiding principle for the construction of quantum operators are the conformal symmetry and infinite dimensional translation symmetry generated by $\phi^{\prime}(x)$. A semi-direct product of these symmetry groups is provided by the Poisson bracket (2.43), which quantum mechanically admits a deformation of the central term. This implies a deformation of the coefficient in front of the linear term in the energy-momentum tensor (2.42)

$$
T(x)=\phi^{\prime 2}(x)-\eta \phi^{\prime \prime}(x) .
$$

The related Virasoro generators satisfy the standard commutation relations with the central charge $c=1+12 \pi \eta^{2} / \hbar$. The deformation parameter $\eta$ is fixed by conformal properties of free-field exponentials. Using the decomposition $\phi(x)=\phi_{0}(x)+\phi_{+}(x)+\phi_{-}(x)$, with

$$
\phi_{0}(x)=\frac{q}{2 \pi}+\frac{p x}{2}, \quad \phi_{+}(x)=-\frac{i}{\sqrt{4 \pi}} \sum_{n>0} \frac{a_{n}^{*}}{n} e^{i n x}, \quad \phi_{-}(x)=\frac{i}{\sqrt{4 \pi}} \sum_{n>0} \frac{a_{n}}{n} e^{-i n x},
$$

a free-field exponential is introduced in a standard normal ordered form

$$
e^{2 \lambda \phi(x)}=e^{2 \lambda \phi_{0}(x)} e^{2 \lambda \phi_{+}(x)} e^{2 \lambda \phi_{-}(x)} .
$$

Requiring unit conformal weight of $e^{2 \phi(x)}$, one finds $\eta=1+b^{2}$, with $2 \pi b^{2}=\hbar$.

Our aim is to construct the vertex operator corresponding to the Liouville exponential (2.44). Building blocks for this construction are the chiral operators

$$
\begin{aligned}
& \psi(x)=e^{-\phi(x)} \\
& A_{p}(x)=\int_{0}^{2 \pi} d z e^{2 \phi_{0}(x+z)-\pi p} e^{2 \phi_{+}(x+z)} e^{2 \phi_{-}(x+z)}, \\
& \chi(x)=\psi(x) A_{p}(x) .
\end{aligned}
$$

The operators $\psi(x)$ and $A_{p}(x)$ are obviously hermitian and the $p$-dependent shift of $\phi_{0}$ in (3.3) is motivated by hermiticity of $\chi(x)$ (see (C.9)). The unit conformal weight of $e^{2 \phi(x)}$ provides zero conformal weight of $A_{p}(x)$ and, therefore the conformal weights of the operators $\psi$ and $\chi$ are the same, like in the classical case. Exchange relations of these operators and their classical counterparts are derived in Appendix C. It is important to note that these relations for the $\psi$ and $\chi$ fields are the same

$$
\begin{aligned}
& \psi(x) \psi(y)=e^{-i(\hbar / 4) \epsilon(x-y)} \psi(y) \psi(x), \\
& \chi(x) \chi(y)=e^{-i(\hbar / 4) \epsilon(x-y)} \chi(y) \chi(x) .
\end{aligned}
$$

Based on (2.44), we are looking for the vertex operator $V$ in the form

$$
V(x, \bar{x})=e^{-i(\hbar / 8)}\left[\psi(\bar{x}) \psi(x)+B_{p} \psi(\bar{x}) \chi(x)+C_{p} \chi(\bar{x}) \psi(x)+D_{p} \chi(\bar{x}) \chi(x)\right],
$$

with $p$-dependent coefficients $B_{p}, C_{p}$ and $D_{p}$. The phase factor $e^{-i(\hbar / 8)}$ provides hermiticity of the first term of $V$-operator, which corresponds to the $i n$-field exponential. The last term describes the out-field exponential, respectively.

To fix $B_{p}, C_{p}$ and $D_{p}$ we use the conditions of locality and hermiticity

$$
\left[V(\tau+\sigma, \tau-\sigma), V\left(\tau+\sigma^{\prime}, \tau-\sigma^{\prime}\right)\right]=0, \quad V^{*}(x, \bar{x})=V(x, \bar{x}) .
$$


The analysis of these equations can be done effectively with the help of exchange relations between the $\psi$ and $\chi$ operators. There are two kind of exchange relations. The first exchanges the ordering of the arguments $x$ and $y$

$$
\chi(x) \psi(y)=e^{i(\hbar / 4) \epsilon(x-y)}\left[\frac{\sinh (\pi p+i \hbar / 2)}{\sinh \pi p} \psi(y) \chi(x)-i \sin (\hbar / 2) \frac{e^{\pi p \epsilon(x-y)}}{\sinh \pi p} \chi(y) \psi(x)\right],
$$

and another the ordering of $\chi$ and $\psi$ fields

$$
\begin{aligned}
\chi(x) \psi(y)= & e^{i(\hbar / 4) \epsilon(x-y)} \\
& \times\left[\frac{\sinh \pi p}{\sinh (\pi p-i \hbar / 2)} \psi(y) \chi(x)-i \sin (\hbar / 2) \frac{e^{(\pi p-i \hbar / 4) \epsilon(x-y)}}{\sinh (\pi p-i \hbar / 2)} \psi(x) \chi(y)\right] .
\end{aligned}
$$

Applying these relations to (3.7) we obtain a set of equations for the functions $B_{p}, C_{p}, D_{p}$. They relate the values of these coefficients with shifted arguments and we have found the following solution of these equations (see Appendix D)

$$
\begin{aligned}
B_{p} & =m_{b} \frac{l_{b} e^{-(\pi p-i \hbar / 2)}+r_{b}}{2 \sinh \pi p \sinh (\pi p-i \hbar / 2)}, \\
C_{p} & =m_{b} \frac{l_{b} e^{(\pi p+i \hbar / 2)}+r_{b}}{2 \sinh \pi p \sinh (\pi p+i \hbar / 2)}, \\
D_{p} & =\frac{m_{b}^{2}}{4 \sinh \pi p \sinh (\pi p+i \hbar)}\left(1+\frac{l_{b}^{2}+r_{b}^{2}+2 l_{b} r_{b} \cosh (\pi p+i \hbar / 2)}{\sinh ^{2}(\pi p+i \hbar / 2)}\right) .
\end{aligned}
$$

The parameters $m_{b}, l_{b}$ and $r_{b}$ arise in the solution as $p$ independent constants. Comparing these expressions with their classical analogs (2.45), we find a naturally interpretation of $m_{b}$ and $\left(l_{b}, r_{b}\right)$ as a renormalized mass and renormalized boundary parameters, respectively.

To cover parabolic and elliptic monodromies, one has to investigate analytical properties (in the variables $\left.p, l_{b}, r_{b}\right)$ of the vertex operator $V$. Work in this direction is in progress.

\section{Conclusions}

For Minkowski space Liouville theory on the strip we have performed a complete analysis of classical solutions regular in the bulk of the strip. These solutions, falling into conformal coadjoint orbits of the energy-momentum tensor [10], can be parameterized by the constant energy density $T_{0}$ of the lowest energy solution in the orbit and an element $\xi(x)$ of the conformal group of the strip.

Depending on the parameters $l$ and $r$, describing the conformally invariant generalized Neumann boundary conditions (FZZT branes) on the left and right boundary of the strip, the solutions have elliptic, parabolic or hyperbolic monodromies. Avoiding singularities in the bulk requires $l, r \geq-1$. Solutions with elliptic monodromy correspond to bound states, those with hyperbolic monodromy to scattering states. For $l+r>0$ all positive values of $T_{0}$ are allowed, the monodromy is then always hyperbolic. For $l+r<0$ negative energies above a threshold depending on $l, r$ and elliptic monodromy are allowed as well as all positive energies and hyperbolic monodromy. The peculiarities of zero energy and parabolic monodromy have been touched, too.

For $l$ or $r=-1$ and certain related $T_{0}$ the Liouville field develops a controlled singularity on the boundaries, just realizing a Dirichlet condition (ZZ brane).

For the Hamilton description of the system the Poisson brackets and the canonical two form has been expressed in terms of the variables $T_{0}$ and $\xi(x)$. To prepare the system for quantization 
an alternative description in terms of a free field has been given, similar to the corresponding construction for the Liouville field theory on a cylinder [11, 12, 13, 14, 15].

We could get a first estimate of quantum effects by discussing semi-classical Bohr-Sommerfeld quantization. The bound state energy levels become quantized and the spectrum agrees with the corresponding quasiclassical limit of the spectrum gained in [3] by conformal bootstrap techniques in Euclidean space.

Finally we have constructed the quantum version of the degenerated exponential of the Liouville field $e^{-\varphi}$. The quantum deformation of the weights in its representation in terms of free field exponentials has been fixed by requiring locality and hermiticity.

There is an obvious schedule for further investigations. From the free field representation of $e^{-\varphi}$ in the hyperbolic sector one can read off the reflection amplitude. Its poles should give information on the full quantum bound state spectrum. With the quantum $e^{-\varphi}$ at hand one can construct generic correlation functions following the technique used for the periodic case [17]. We also hope to fully explore the limiting ZZ case within the canonical quantization.

\section{A Calculation of 2-forms}

\section{A.1 Dirichlet condition}

Equation (2.15) leads to the following parametrization of the canonical coordinates

$$
\begin{aligned}
& \varphi(\tau, \sigma)=\frac{1}{2} \log \xi^{\prime}(x) \xi^{\prime}(\bar{x})-\log \sin \frac{1}{2}[\xi(x)-\xi(\bar{x})]-\log 2 m, \\
& \pi(\tau, \sigma)=\frac{\xi^{\prime \prime}(x)}{2 \xi^{\prime}(x)}+\frac{\xi^{\prime \prime}(\bar{x})}{2 \xi^{\prime}(\bar{x})}-\frac{\xi^{\prime}(x)-\xi^{\prime}(\bar{x})}{2} \cot \frac{1}{2}[\xi(x)-\xi(\bar{x})] .
\end{aligned}
$$

The canonical form $(2.23)$ can be represented in the form $\omega=\omega_{0}+\bar{\omega}_{0}+\omega_{1}$, where

$$
\begin{aligned}
& \omega_{0}=\frac{1}{4} \int_{0}^{\pi} d \sigma\left[\frac{\delta \xi^{\prime \prime}(x) \wedge \delta \xi^{\prime}(x)}{\xi^{\prime 2}(x)}-\delta \xi^{\prime}(x) \wedge \delta \xi(x)\right], \\
& \bar{\omega}_{0}=\frac{1}{4} \int_{0}^{\pi} d \sigma\left[\frac{\delta \xi^{\prime \prime}(\bar{x}) \wedge \delta \xi^{\prime}(\bar{x})}{\xi^{\prime 2}(\bar{x})}-\delta \xi^{\prime}(\bar{x}) \wedge \delta \xi(\bar{x})\right],
\end{aligned}
$$

while $\omega_{1}$ turns to a boundary term, since it is represented as an integral from a derivative by $\sigma$. The 2 -form $\omega_{1}$ vanishes due to the monodromy properties of $\xi$. Using the doubling trick as in $(2.19)$, we rewrite the sum $\omega_{0}+\bar{\omega}_{0}$ into $(2.24)$.

\section{A.2 Neumann conditions}

The general solution (2.32) can be written in the standard Liouville form

$$
V=\frac{1+m^{2} F(x) \bar{F}(\bar{x})}{\sqrt{F^{\prime}(x) \bar{F}^{\prime}(\bar{x})}},
$$

with

$$
F(x)=\frac{u e^{p \xi(x)}+l e^{\pi p}+r}{2 \sinh \pi p}, \quad \bar{F}(\bar{x})=\frac{u e^{p \xi(x)}+l e^{\pi p}+r}{2 \sinh \pi p} .
$$

Applying the same technique as before, we express the canonical form (2.23) in terms of parameterizing $F$ and $\bar{F}$ fields

$$
\omega=\frac{1}{4} \int_{0}^{\pi} d \sigma\left[\frac{\delta F^{\prime \prime}(x) \wedge \delta F^{\prime}(x)}{F^{\prime 2}(x)}+\frac{\delta \bar{F}^{\prime \prime}(\bar{x}) \wedge \delta \bar{F}^{\prime}(\bar{x})}{\bar{F}^{\prime 2}(\bar{x})}\right]+\text { B.T. }
$$


with the boundary term

$$
\text { B.T. }=\frac{\delta F^{\prime}(x) \wedge \delta \bar{F}^{\prime}(\bar{x})}{4 F^{\prime}(x) \bar{F}^{\prime}(\bar{x})}-\frac{1}{2} \delta \log \left(\frac{F^{\prime}(x)}{\bar{F}^{\prime}(\bar{x})}\right) \wedge \delta \log \left(1+m^{2} F(x) \bar{F}(\bar{x})\right)+\left.\frac{\delta F(x) \wedge \delta \bar{F}(\bar{x})}{1+m^{2} F(x) \bar{F}(\bar{x})}\right|_{0} ^{\pi} .
$$

Then, using (A.1) and the monodromy properties of $\xi$-field we get (2.35).

\section{B Comparison of quasiclassical quantization with the corresponding limit of the conformal bootstrap spectrum}

First we write our formula (2.30) for $\theta_{*}(l, r)$ in a form more suitable for the comparison with [3]. Denoting $l=l_{1}, r=l_{2}$ and defining $\vartheta_{j}$ in $(0, \pi)$ for $\left|l_{j}\right|<1$ by

$$
l_{j}=\cos \vartheta_{j}
$$

we get

$$
\theta_{*}(l, r)=\frac{\vartheta_{1}+\vartheta_{2}}{\pi}-1 .
$$

This brings (2.38) in the form

$$
\left(T_{0}\right)_{n}=-\frac{\hbar^{2}}{4 \pi^{2}} n^{2}+\frac{\hbar}{2 \pi}\left(\frac{\vartheta_{1}+\vartheta_{2}}{\pi}-1\right) n-\frac{\left(\vartheta_{1}+\vartheta_{2}\right)^{2}}{4 \pi^{2}}+\frac{\vartheta_{1}+\vartheta_{2}}{2 \pi}-\frac{1}{4} .
$$

The dictionary to compare our normalizations of the Liouville field, the mass and boundary parameters $\varphi, m, l_{j}$ with that of $[3]\left(\phi_{T}, \mu, \rho_{j}\right)$ is

$$
\varphi=b \phi_{T}, \quad m^{2}=\mu \pi b^{2}, \quad l_{j}=\sqrt{\frac{\pi}{\mu}} b \rho_{j} .
$$

According to [3], the state space of Liouville theory on the strip is the direct sum of highest weight $\left(\Delta_{\beta}=\beta(Q-\beta), Q=1 / b+b\right)$ representations of the Virasoro algebra. There is a continuum contribution $\beta \in Q / 2+i \mathbb{R}^{+}$, and depending on the boundary parameters a discrete contribution [3] characterized by

$$
\beta=Q-\left|\sigma_{ \pm}\right|+n b+\hat{n} \frac{1}{b}<\frac{Q}{2},
$$

where $n, \hat{n}$ are non-negative integers and $\sigma_{ \pm}=i\left(s_{2} \pm s_{1}\right)$ with

$$
\cosh \left(2 \pi b s_{j}\right)=\frac{\rho_{j}}{\sqrt{\mu}} \sqrt{\sin \left(\pi b^{2}\right)}
$$

The evaluation of (B.3) in the quasiclassical limit $b \rightarrow 0$ expressed in our boundary parameters $l_{j}$ (for $\left|l_{j}\right|<1$ ) gives

$$
s_{j}=i \frac{\arccos l_{j}}{2 \pi b}+\mathcal{O}\left(b^{2}\right) .
$$

Inserting this into (B.2) one first notices that for small enough $b$ the option $\hat{n} \neq 0$ is switched off. On top of this, in this limit only the choices $\sigma_{+}$and $\arccos l_{j} \in(0, \pi)$ obey the inequality in (B.2). Altogether this leads to

$$
b^{2} \Delta_{n}=-b^{4} n(n+1)+b^{2}\left(\frac{\vartheta_{1}+\vartheta_{2}}{\pi}-1\right) n-\frac{\left(\vartheta_{1}+\vartheta_{2}\right)^{2}}{4 \pi^{2}}+\frac{\vartheta_{1}+\vartheta_{2}}{2 \pi} .
$$


After the identifications $\hbar=2 \pi b^{2}$ and $\left(T_{0}\right)_{n}=b^{2} \Delta_{n}$ this agrees with (B.1) up to the trivial shift by $-1 / 4$ and the replacement $n(n+1) \mapsto n^{2}$, valid for large $n$ and common for the quasiclassical approximation. The continuous spectrum in [3] corresponds to the our solutions with hyperbolic monodromy.

Although not touching the issue of quantization for the Dirichlet case in this paper, we nevertheless can add already one interesting observation concerning the spectrum of $T_{0}$. From Subsection 2.1 we know that classically there is only one value for $T_{0}$ allowed. It is $T_{0}=-1 / 4$, if on both sides of the strip Dirichlet conditions are imposed, and $T_{0}=-(\arccos r)^{2} /\left(4 \pi^{2}\right)$ for Dirichlet on the left and generalized Neumann with parameter $r$ on the right. With the just derived translation rule $T_{0}=b^{2} \Delta-1 / 4$ this corresponds to the conformal dimensions of highest weight states of the contributing Verma modules $\Delta=0$ and $\Delta=s^{2}+1 /\left(4 b^{2}\right)$, respectively. This agrees in leading order of $b$ with the full quantum result via conformal bootstrap reported in $[1,18]$ for the $(1,1) \mathrm{ZZ}$ brane. Note that $s_{j}$ defined according to [3] in our equation (B.4) differs by a factor $1 / 2$ from $s$ in $[1,18]$.

\section{Exchange relations}

\section{C.1 Poisson brackets algebra of chiral fields}

In this appendix we use the method applied in [19]. The chiral field $\psi(x)=e^{-\phi(x)}$ is the classical analog of the operator (3.2) and the canonical Poisson brackets (2.41) are equivalent to

$$
\{\psi(x), \psi(y)\}=\frac{1}{4} \epsilon(x-y) \psi(x) \psi(y),
$$

which quantum mechanically becomes (3.5).

The operator (3.3) corresponds to the field $A_{p}(x)=\int_{0}^{2 \pi} d z e^{2 \phi(y+z)-\pi p}$ and its Poisson bracket with the $\psi$-field reads

$$
\left\{\psi(x), A_{p}(y)\right\}=-\frac{1}{2} \psi(x) \int_{0}^{2 \pi} d z e^{2 \phi(y+z)-\pi p}(\epsilon(x-y-z)+1) .
$$

Due to the stair-step character of the $\epsilon$-function the following identity holds

$$
\epsilon(a+b)-\epsilon(a)-\epsilon(b)= \pm 1,
$$

and since $\cosh \pi p \pm \sinh \pi p=e^{ \pm \pi p}$, we find

$$
\epsilon(x-y-z)=\epsilon(x-y)-\epsilon(z)-\frac{\cosh \pi p}{\sinh \pi p}+\frac{e^{\pi p[\epsilon(x-y-z)-\epsilon(x-y)+\epsilon(z)]}}{\sinh \pi p} .
$$

Inserting (C.4) into (C.2) and using that the function $2 \phi(y+z)+\pi p \epsilon(x-y-z)$ is periodic in $z$, we can shift the integration domain in the last term and obtain

$$
\left\{\psi(x), A_{p}(y)\right\}=\frac{1}{2}\left(\frac{\cosh \pi p}{\sinh \pi p}-\epsilon(x-y)\right) \psi(x) A_{p}(y)-\frac{e^{-\pi p \epsilon(x-y)}}{2 \sinh \pi p} \psi(x) A_{p}(x) .
$$

By (C.1) and (C.5) the field $\chi(x)=\psi(x) A_{p}(x)$ satisfies the relation

$$
\{\psi(x), \chi(y)\}=\frac{1}{2}\left(\frac{\cosh \pi p}{\sinh \pi p}-\frac{1}{2} \epsilon(x-y)\right) \psi(x) \chi(y)-\frac{e^{-\pi p \epsilon(x-y)}}{2 \sinh \pi p} \chi(x) \psi(y) .
$$

To find a closed form of the Poisson brackets

$$
\left\{A_{p}(x), A_{p}(y)\right\}=\int_{0}^{2 \pi} d z e^{2 \phi(x+z)-\pi p} e^{2 \phi\left(y+z^{\prime}\right)-\pi p} \epsilon\left(x-y+z-z^{\prime}\right)
$$


in terms of the $A_{p}$-field, we use the identity

$$
\begin{aligned}
\epsilon(x- & \left.y+z-z^{\prime}\right)=\epsilon(x-y)+\epsilon(z)-\epsilon\left(z^{\prime}\right) \\
& +\frac{e^{-\pi p \epsilon(x-y)}}{\sinh \pi p} e^{\pi p\left[\epsilon\left(x-y-z^{\prime}\right)+\epsilon\left(z^{\prime}\right)\right]}-\frac{e^{\pi p \epsilon(x-y)}}{2 \sinh \pi p} e^{-\pi p[\epsilon(x-y+z)-\epsilon(z)]} \\
& +\frac{e^{\pi p \epsilon\left(z^{\prime}\right)}}{2 \sinh \pi p} e^{\pi p\left[\epsilon\left(x-y+z-z^{\prime}\right)-\epsilon(x-y+z)\right]}-\frac{e^{\pi p \epsilon(z)}}{2 \sinh \pi p} e^{-\pi p\left[\epsilon\left(x-y+z-z^{\prime}\right)-\epsilon\left(x-y-z^{\prime}\right)\right]},
\end{aligned}
$$

which follows from (C.4). The contributions of the last two terms of (C.7) in the integral (C.6) cancel each other and provide the result

$$
\left\{A_{p}(x), A_{p}(y)\right\}=\epsilon(x-y) A_{p}(x) A_{p}(y)+\frac{e^{-\pi p \epsilon(x-y)}}{2 \sinh \pi p} A_{p}^{2}(x)-\frac{e^{\pi p \epsilon(x-y)}}{2 \sinh \pi p} A_{p}^{2}(y) .
$$

The calculation of the Poisson brackets between $\chi$-fields is now straightforward and we end up with

$$
\{\chi(x), \chi(y)\}=\frac{1}{4} \epsilon(x-y) \chi(x) \chi(y)
$$

which indicates that the fields $\psi$ and $\chi$ are related canonically.

\section{C.2 Operator algebra}

First note that the exchange relations of $q$-exponentials and $p$-dependent functions is

$$
e^{a q} f(p)=f(p+i a \hbar) e^{a q} .
$$

An intermediate step towards the exchange relations between the $\psi$ and $\chi$ operators is a calculation of the quantum analog of (C.5). Due to (3.1) and (C.8), from (3.2)-(3.3) we have

$$
\psi(x) A_{p}(y)=\int_{0}^{2 \pi} d z e^{2 \phi(y+z)} e^{-(\pi p-i \hbar)} \psi(x) e^{i(\hbar / 2) \epsilon(x-y-z)} .
$$

To rewrite this equation as an exchange relation, we use the identity

$$
\begin{aligned}
\sinh & \pi p e^{i(\hbar / 2)[\epsilon(x-y-z)-\epsilon(x-y)+\epsilon(z)]} \\
& =\sinh (\pi p-i \hbar / 2)+i \sin (\hbar / 2) e^{\pi p[\epsilon(x-y-z)-\epsilon(x-y)+\epsilon(z)]},
\end{aligned}
$$

based on (C.3). Inserting $e^{i(\hbar / 2) \epsilon(x-y-z)}$ from (C.10) into (C.9) we obtain

$$
\begin{aligned}
\psi(x) A_{p}(y)= & e^{i(\hbar / 2) \epsilon(x-y)} \frac{\sinh \pi p}{\sinh (\pi p+i \hbar / 2)} A_{p}(y) \psi(x) \\
& +i \sin (\hbar / 2) \frac{e^{-\pi p \epsilon(x-y)}}{\sinh (\pi p+i \hbar / 2)} A_{p}(x) \psi(x),
\end{aligned}
$$

which for $x=y$ yields

$$
\psi(x) A_{p}(x)=A_{p}(x) \psi(x) .
$$

The derivation of the exchange relation between the $\psi$ and $\chi$ operators (see (3.4)) is now straightforward and we obtain

$$
\psi(x) \chi(y)=e^{i(\hbar / 4) \epsilon(x-y)}\left[\frac{\sinh (\pi p-i \hbar / 2)}{\sinh \pi p} \chi(y) \psi(x)+i \sin (\hbar / 2) \frac{e^{-\pi p \epsilon(x-y)}}{\sinh \pi p} \psi(y) \chi(x)\right] .
$$


The exchange relation (3.8) is derived in a similar way and (3.9) follows from (C.11) and (3.8) by simple algebraic manipulations.

The next step is the exchange relation between the $A_{p}$-operators, which is obtained in the same manner and in a symmetrized form it reads

$$
\begin{aligned}
A_{p}(x) & A_{p}(y) e^{-i(\hbar / 2) \epsilon(x-y)}-A_{p}(y) A_{p}(x) e^{i(\hbar / 2) \epsilon(x-y)} \\
= & i \sin (\hbar / 2)\left(\frac{e^{(\pi p+i \hbar) \epsilon(x-y)}}{\sinh (\pi p+i \hbar)} A_{p}^{2}(y)-\frac{e^{-(\pi p+i \hbar) \epsilon(x-y)}}{\sinh (\pi p+i \hbar)} A_{p}^{2}(x)\right) .
\end{aligned}
$$

This finally provides (3.6).

\section{Locality and Hermiticity of $V$-operator}

The locality condition (3.7) is equivalent to the symmetry of the product $V(\sigma,-\sigma) V\left(\sigma^{\prime},-\sigma^{\prime}\right)$ under $\sigma \leftrightarrow \sigma^{\prime}$. Let us collect the terms with a given power $N$ of the $\chi$-field. The number $N$ changes from 0 to 4 . There is only one term with $N=0$

$$
C_{\sigma, \sigma^{\prime}}=e^{-i \hbar / 4} \psi(-\sigma) \psi(\sigma) \psi\left(-\sigma^{\prime}\right) \psi\left(\sigma^{\prime}\right),
$$

which is symmetric due to (3.5). The case $N=4$ is similar because of (3.6).

For the terms with $N=1$ we use the exchange relation (3.9), moving the $\chi$-field in each term to the right hand side. Replacing then $\chi$ by $\psi A_{p}$, we find the following structure

$$
\Lambda_{p}^{(1)} C_{\sigma, \sigma^{\prime}} A_{p}(\sigma)+\Lambda_{p}^{(2)} C_{\sigma, \sigma^{\prime}} A_{p}(-\sigma)+\Lambda_{p}^{(3)} C_{\sigma, \sigma^{\prime}} A_{p}\left(\sigma^{\prime}\right)+\Lambda_{p}^{(4)} C_{\sigma, \sigma^{\prime}} A_{p}\left(-\sigma^{\prime}\right) .
$$

with $p$ dependent coefficients $\Lambda_{p}^{(1)}, \ldots, \Lambda_{p}^{(4)}$. The symmetry of (D.1) requires $\Lambda_{p}^{(1)}=\Lambda_{p}^{(3)}$ and $\Lambda_{p}^{(2)}=\Lambda_{p}^{(4)}$. These conditions lead to the equations

$$
\begin{aligned}
B_{p-i \hbar / \pi} & =\frac{e^{i(\hbar / 2)}}{\sinh (\pi p-i \hbar)}\left[\frac{\sinh (\pi p-i \hbar / 2)}{\sinh (\pi p-3 i \hbar / 2)} B_{p}+i \frac{e^{-\pi p} \sin (\hbar / 2)}{\sinh (\pi p-3 i \hbar / 2)} C_{p}\right], \\
C_{p-i \hbar / \pi} & =i \frac{\sin (\hbar / 2) e^{\pi p-i \hbar / 2}}{\sinh (\pi p-i \hbar)} B_{p}+\frac{e^{-i(\hbar / 2)} \sinh (\pi p+i \hbar / 2)}{\sinh (\pi p-i \hbar)} C_{p},
\end{aligned}
$$

which are simplified for the linear combinations

$$
\begin{aligned}
& X_{p}=\sinh (\pi p+i \hbar / 2) C_{p}-\sinh (\pi p-i \hbar / 2) B_{p}, \\
& Y_{p}=-e^{-\pi p} \sinh (\pi p+i \hbar / 2) C_{p}+e^{\pi p} \sinh (\pi p-i \hbar / 2) B_{p},
\end{aligned}
$$

in the form

$$
X_{p-i \hbar / \pi}=X_{p}, \quad Y_{p-i \hbar / \pi}=Y_{p} .
$$

Thus, with $X_{p}=2 L$ and $Y_{p}=2 R$, where $L$ and $R$ are arbitrary complex numbers, we find

$$
B_{p}=\frac{L e^{-\pi p}+R}{\sinh \pi p \sinh (\pi p-i \hbar / 2)}, \quad C_{p}=\frac{L e^{\pi p}+R}{\sinh \pi p \sinh (\pi p+i \hbar / 2)} .
$$

The hermiticity condition (3.7) puts restrictions on the parameters $L$ and $R$. Making use of the exchange relations (C.11) and (3.8), one finds a relation between $B_{p}$ and $C_{p}$ and their complex conjugates, reducing the freedom of two complex parameters to two real ones. With an additional free real parameter from $D_{p}$ we finally obtain with real $l_{b}, r_{b}$ and $m_{b}(3.10)$ and (3.11).

Due to the symmetry between the $\psi$ and $\chi$ fields, the case $N=3$ gives the same result as $N=1$.

The analysis of the case $N=2$ can be done similarly, but now with the known $B_{p}, C_{p}$ and for $D_{p}$ we end up with (3.12). 


\section{Acknowledgements}

We thank Cosmas Zachos for helpful discussions. G.J. is grateful to the organizers of "The O'Raifeartaigh Symposium" for the invitation. He thanks Humboldt University, AEI Golm, ICTP Trieste and ANL Argonne for hospitality, where a main part of his work was done. His research was supported by grants from the DFG (436 GEO 17/3/06) and GRDF (GEP1-3327TB-03). H.D. was supported in part by DFG with the grant DO 447-3/3.

\section{References}

[1] Zamolodchikov A.B., Zamolodchikov Al.B., Liouville field theory on a pseudosphere, hep-th/0101152.

[2] Fateev V., Zamolodchikov A.B., Zamolodchikov Al.B., Boundary Liouville field theory. I. Boundary state and boundary two-point function, hep-th/0001012.

[3] Teschner J., Remarks on Liouville theory with boundary, hep-th/0009138.

[4] Martinec E.J., The annular report on non-critical string theory, hep-th/0305148.

[5] Gervais J.L., Neveu A., The dual string spectrum in Polyakov's quantization. I, Nuclear Phys. B 199 (1982), 59-76.

[6] Gervais J.L., Neveu A., Dual string spectrum in Polyakov's quantization. II. Mode separation, Nuclear Phys. B 209 (1982), 125-145.

[7] Gervais J.L., Neveu A., Novel triangle relation and absence of tachyons in Liouville string field theory, Nuclear Phys. B 238 (1984), 125-141.

[8] Gervais J.L., Neveu A., Green functions and scattering amplitudes in Liouville string field theory. I, Nuclear Phys. B 238 (1984), 396-406.

[9] Cremmer E., Gervais J.L., The quantum strip: Liouville theory for open strings, Comm. Math. Phys. 144 (1992), 279-302.

[10] Balog J., Feher L., Palla L., Coadjoint orbits of the Virasoro algebra and the global Liouville equation, Internat. J. Modern Phys. A 13 (1998), 315-362, hep-th/9703045.

[11] Curtright T.L., Thorn C.B., Conformally invariant quantization of the Liouville theory, Phys. Rev. Lett. 48 (1982), 1309-1313, Erratum, Phys. Rev. Lett. 48 (1982), 1768-1768.

[12] Braaten E, Curtright T.L., Thorn C.B., An exact operator solution of the quantum Liouville field theory, Ann. Physics 147 (1983), 365-416.

[13] Otto H.J., Weigt G., Construction of exponential Liouville field operators for closed string models, Z. Phys. C 31 (1986), 219-228.

[14] Teschner J., Liouville theory revisited, Classical Quantum Gravity 18 (2001), 153-222, hep-th/0104158.

[15] Jorjadze G., Weigt G., Poisson structure and Moyal quantisation of the Liouville theory, Nuclear Phys. B 619 (2001), 232-256, hep-th/0105306.

[16] Alekseev A., Shatashvili S.L., From geometric quantization to conformal field theory, Comm. Math. Phys. 128 (1990), 197-212.

[17] Jorjadze G., Weigt G., Correlation functions and vertex operators of Liouville theory, Phys. Lett. B 581 (2004), 133-140, hep-th/0311202.

[18] Nakayama Y., Liouville field theory: A decade after the revolution, Internat. J. Modern Phys. A 19 (2004), 2771-2930, hep-th/0402009.

[19] Ford C., Jorjadze G., A causal algebra for Liouville exponentials, Classical Quantum Gravity 23 (2006), 6007-6014, hep-th/0512018. 